\title{
Advancements in simulations for evacuation, hazardous chemical exposure and active shooter engagements
}

\author{
J. L. Smith
}

Applied Research Associates, Inc., USA

\begin{abstract}
Recent terrorist attacks in Mumbai and tragedies such as the Virginia Tech massacre underscore the critical need to understand and train for active shooter and force-on-force engagements with adversary forces. Terrorist organizations are known to possess the intent and capability to attack highly secure critical facilities such as nuclear storage and power facilities as well as schools, stadiums, and other public gathering places. Regardless of whether a disaster is man-made such as a bombing or active shooter event, or naturally occurring such as a tornado, evacuation of people away from the danger is of paramount importance. Advanced agent-based modeling and simulation have evolved to enable virtual investigation of such scenarios. This paper offers insight into the benefits and use of advanced science-based tools to investigate these scenarios in-depth. Virtual simulations provide cost-effective, valuable insight into scenarios that cannot be realistically played out in real-world exercises.

Keywords: emergency evacuation, scenario-based simulation, agent-based modeling, active shooter, disaster management, people movement, evacuations, gaming scenarios.
\end{abstract}

\section{Introduction}

In 2007, two separate coordinated attacks on the Virginia Tech campus left 32 dead and countless wounded. In 2008, more than ten coordinated shooting and bombing attacks across Mumbai India killed 173, wounding more than 308. An armed guard was shot dead in July of 2009 in front of America's Holocaust Museum. On November 5, 2009 twelve US soldiers were killed and 31 others wounded in the radical shooting at Fort Hood, TX. Many other events, including 
the Pentagon shooting, the Washington DC sniper, hospital shootings Connecticut and Tennessee, and the attempted assassination of Congresswoman Giffords have left even more Americans killed or severely wounded. The common thread: all involved armed shooters; all shooters successfully executed their attacks. Clearly, armed attacks are a matter of extreme concern to world leaders and an area that deserves more focus and analysis so that we may be better prepared to defend ourselves. Whether the perpetrators are radical extremists on a shooting rampage, emotionally distressed individuals seeking vengeance, or skilled and trained terrorists with a specific political goal, these events only become more frequent as time progresses, encouraging even more attacks. Never has it been more critical for our society to understand, plan and train responsibly for active shooter and force-on-force engagements.

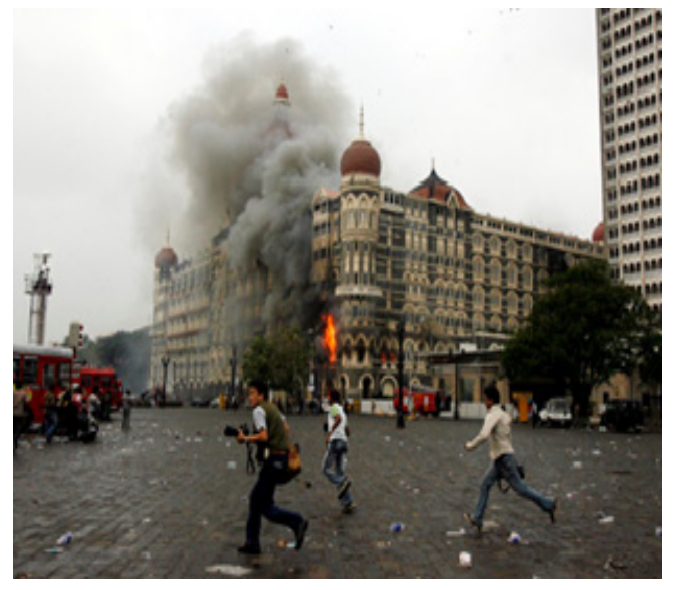

Figure 1: Mumbai style attacks, active shooters, terrorist attacks on soft targets. How can we prepare?

Furthermore, evacuating large crowds of people under any circumstance is a challenge. Evacuation of large facilities during an emergency or disaster is a much more complex task because of the added elements of chaos, panic, and the high density of the population. As terrorists move more towards preying on soft targets such as hotels, hospitals, airports, train stations and stadiums, the potential for large government, commercial, residential and sports facilities to become targets is a reality for which we must prepare. Non-terror related events and accidents involving inadequate planning and training for mass evacuations are also all too common. Examples include the February 2003 Rhode Island nightclub fire that killed at least 96 people and the August 2, 2004 supermarket fire in Asuncion, Paraguay, that killed over 300 shoppers. Determining the most effective evacuation plan for a large public facility requires in-depth analysis of multiple factors. Evaluating the best routes, foreseeing potential problems, addressing the chaos/panic factor, and orchestrating the evacuation are all critical aspects that should be evaluated in a well developed disaster management plan. 
While many facility owners do conduct evacuation drills, such drills are most often not accomplished during times of peak facility occupancy and do not include event based scenarios such as a bombing followed by an active shooter, fire, smoke, partial collapse and falling debris that would impair or preclude egress through various areas of the facility. Until recently, many evacuation plans called for phased evacuations of only affected floors or areas of large facilities (e.g., if a fire was detected in a high rise building, that floor plus the adjacent floors would be evacuated first followed by other areas). Hence, it has to date been difficult to accurately understand and predict full building evacuations under realistic emergency conditions. Mass or total building evacuations pose additional problems of stairwell congestion, chaos and potential stampeding of panicked occupants. This is even more important in the post-September $11^{\text {th }}$ era where most people, especially in high rise facilities, now seem to demonstrate a higher propensity to evacuate quickly.

Typical mass-gathering establishments such as schools, stadiums, hotels, highly secure critical facilities including nuclear storage, power and military facilities, or symbolic icons are all extremely attractive targets for terrorist organizations. Until recently, it was not possible to obtain detailed post-mortem analyses for these armed attack scenarios prior to an actual attack. Occupants and responders were left to defend themselves with traditional evacuation procedures and guard force engagements, then hope for the best. Scientists and analysts have however made tremendous strides with recent advancements in modeling and simulation methods which now allow us to conduct detailed virtual investigations of such scenarios before the event even occurs. The best way to prepare for an event without directly experiencing it is to accurately create a multivariate model of the attack scenario, analyze your response plans within the model, and evaluate the results to identify the most appropriate response. New advancements and emerging technologies will assist us in preparing highly effective mitigation and response plans to better prepare and defend our citizens from these devastating active-shooter attacks, bombings and other disasters.

\section{Agent-based modeling and simulation}

In an instant, terror events can create an environment of chaos with an urgent need to effectively and efficiently remove people from harm's way, particularly during prolonged active shooter and force-on-force engagements such as the 2008 Mumbai attacks. To truly prepare for such events, traditional table-top exercises, full-scale re-enactments of attacks, and other training events are based on very limited assumptions as to the adversary's behavior, decision-making ability, skill level, intentions, and level of knowledge of the facility or operation. These assumptions, historical precedence, and other training methods can never cover every possible "what-if" scenario, making it difficult for emergency planners and leaders to obtain useful and realistic data. Thus, training exercises and traditional methods of preparedness are only effective if the adversary plays by the rules of the scenario. These methods of preparing have historically left our defenders and our victims highly vulnerable to the unpredictable and 
innovative approaches of armed shooters. However, advanced modeling and simulation can now provide a more objective view and take into account a multitude of independent variables which serve as critical tools in planning effective response and recovery operations to save human lives.

When preparing for these events, there is much more to consider than simply deploying security forces and evacuating individuals through the nearest exits. The complexity of these attacks and the numerous support functions required to respond successfully demand that we know critical details such as the next probable step of the aggressors, the most effective method of neutralizing the threat; the number of casualties and fatalities that may occur, the number of security/police forces, first responders and ambulances required for effective response, and the estimated quantity of hospital beds and medical staff required for treating casualties resulting from the event. Such detailed data for these complex scenarios is virtually impossible to obtain solely through routine drills, traditional tabletop exercises, typical flow-based pedestrian modeling, historic precedence or other training events.

Until recently, analytic methods for modeling and simulation of masscasualty events (such as an active shooter) have been lacking sufficient fidelity and realism to successfully evaluate such levels of complexity required for these scenarios. For example, a popular model typically used for mass movement of individuals during an evacuation is the hydraulic model, which essentially treats the mass movement of people as a flowing fluid, moving uniformly and constantly to specific destinations at the same rate. However, these fluid-based models have many flaws when it comes to realistically replicating pedestrian evacuations during an event, including the assumption that all people will start moving at the same time, and that everyone will move uniformly, including speed and direction. Such models cannot take into account the chaotic environment, the differences in human behavior, emotional states, cognitive decision-making processes or personal knowledge of the facility. Similarly, typical video-game style attack models (such as shootings) are based on scripted outcomes, following an underlying framework of "if, then" rules that define the attacker's actions. These models do not allow for the differences of behavior in attackers of varying decision-making ability, their motives, their levels of insider knowledge, and leave emergency responders with very limited information that is useful or applicable to real life scenarios.

Advances in state of the art modeling techniques and cognitive task analysis science have facilitated the development of agent-based modeling and simulation applications. These agent-based applications represent the next generation of mass-casualty modeling and simulation analysis, where each individual is recognized as its own unique autonomous agent with its own distinctive set of characteristics and knowledge base. Figure 2 shows a selection from a crowd of these individual agents, each moving and reacting to the three armed shooters according to their own cognition. Using agent-based modeling, planners can quickly assess a range of attack scenarios with unique attributes for the aggressors, responders, facility and specialized targets within the facility. This approach enables responders to visually witness countless "what if" scenarios 
through to completion, adjust their response techniques or methods, and view the new outcomes to analyze their effectiveness. Everything from response force entry locations to adversary cognition; from accurate crowd response to local medical support requirements are included in unprecedented levels of fidelity. This new modeling approach, used by programs such as the event simulation tool ESIM (Event SIMulator developed by Applied Research Associates (ARA)), even includes the ability of individual agents to practice situational awareness and share learned knowledge about the facility/event with other agents they encounter throughout the scenario.

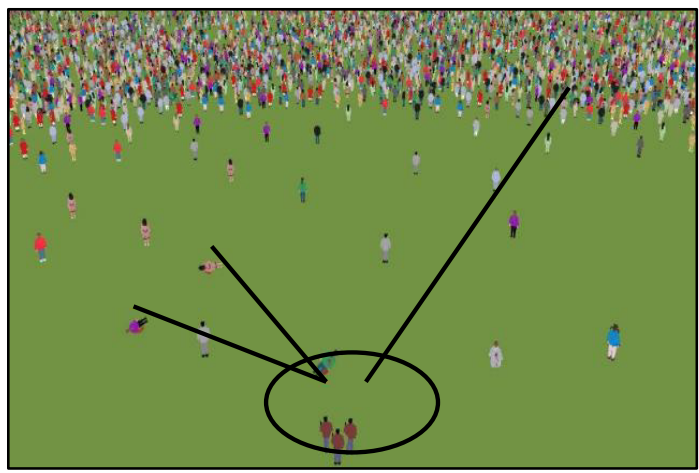

Figure 2: Human behavior in crowds attacked by armed shooters provides keen insight for creating plans, casualty estimates, and evacuation realities.

Development of such applications requires extensive involvement of not only software developers, mathematicians and scientists, but also cognitive psychologists who address idiosyncrasies in human behavior and cognitive decision making ability. In addition to decision making based on deductive reasoning, past experience and specialized knowledge, such models must also consider a range of psychological parameters, such as mental states when people are disoriented, frightened or panicked, or mental distractions such as parents evacuating with their small children. Routine habits can also be captured in agent-based models. For example, people with no direct instruction will typically exit a facility or space through the same outlet that they used to enter, even if there is a closer or more convenient exit nearby. Modeling has already demonstrated how adding simple directed evacuation can drastically improve a crowd's response to an attack and save many lives, despite signage and labeling of exit routes.

New agent-based modeling and simulation tools, such as ESIM, also equip analysts with the ability to maintain the fidelity of the specific facility that is being examined. Figure 3 depicts a screenshot from an active shooter scenario at a customized high-security facility with specific material properties and unique layout. These facility models are realistic, dynamic, and replete with the 


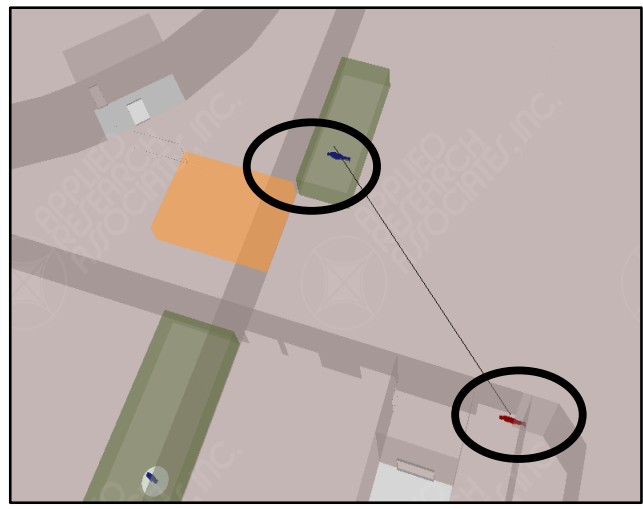

Figure 3: $\quad$ Programmed for high marksmanship skill, an active shooter uses explosives to penetrate a high-security facility and engages an armed responder in a 1-on-1 shooting, leaving both individuals dead.

capabilities necessary to analyze and prepare for a host of attacks or undesirable events.

Using advanced agent-based modeling with cognitive task analysis, it is possible to analyze the progression of assailants through a facility in a hypothetical situation. Analysts are then able to glean critical data from the event and assess multiple possible outcomes based on the effectiveness of a given response to the event. Such information may include:

- Optimal avenues of approach for security/police response forces based on facility layout, situational details, experience/training and capabilities

- Time/rate of progression of the assailants through the facility based on aggressor experience/training, capabilities, knowledge of the facilities and procedures, and planning of the attack

- Optimal evacuation avenues and methods (or shelter in-place positioning) for bystanders or facility occupants

- Delays in facility occupant response to the event such as injury, uncertainty, fear, denial, disability, separation from other occupants, etc.

- Location and vulnerability of potential targets within the facility

- Anticipated interception/neutralization time required by response forces

- Potential 'friendly-fire' incidents based on response force positions and avenues of approach

- Obstacles presented by the facility/event such as construction, unfamiliarity with the facility, smoke, darkness, locked/hardened doors or windows, damaged/impassible corridors, etc. 
- Anticipated number and location of casualties/fatalities

- Number of first responders/ambulances/medical staff/hospital beds required for response and treatment of casualties

- Added benefits provided by directed evacuation of building occupants and shared knowledge throughout the event.

Although this list is not all-inclusive, these few provisions address critical topics that will aid in ensuring effective preparation for response and mitigation of potentially catastrophic attacks.

\subsection{Scenarios and results}

The types of scenarios that may be simulated in an agent-based event/evacuation simulation are numerous. The significant advantage of an advanced simulation tool such as ESIM is the ability to introduce multiple events within a single evacuation scenario that allows the user to evaluate potential real world situations that could not be safely or economically reproduced in an actual evacuation or active shooter drill. ESIM is configured to simulate any combination of two types of events. These are classified as either "environmental" that will slow or impede an agent's progress, or "standard" that will kill all or injure agents within the designated event area and deny all access to others attempting to pass through an area. Examples include (Table 1):

Table 1: $\quad$ Typical types of events considered in ESIM.

\begin{tabular}{ll}
\hline Environmental Event & Standard Event \\
\hline Loss of power & Explosion \\
\hline Loss of light (darkness) & Toxic chemical exposure \\
Water (flooding) & Structural collapse \\
Smoke (light) & Smoke \\
Biological agent exposure & Fire blocking egress \\
& Shooting \\
& Physical Assault \\
\hline
\end{tabular}

\subsection{Example - chemical exposure}

A terrorist release of a toxic material such as Sarin Gas in a highly populated area like a public building during an event is of interest not only to potential terrorists but also to governments and security professionals. In this example, consider a facility in which 5,000 people (men, women and children) are gathered. Some of these people are employees with full knowledge of the facility but the majority are casual visitors. A model was created in which a crude Sarin delivery device was used to introduce the gas in the lobby area. Figure 4 shows the results of the analysis of the spread of this nerve agent over a period of 15 minutes. In this particular case, while there is some gas movement, the majority remains in the general vicinity of the lobby as this area's ventilation system is segmented. 


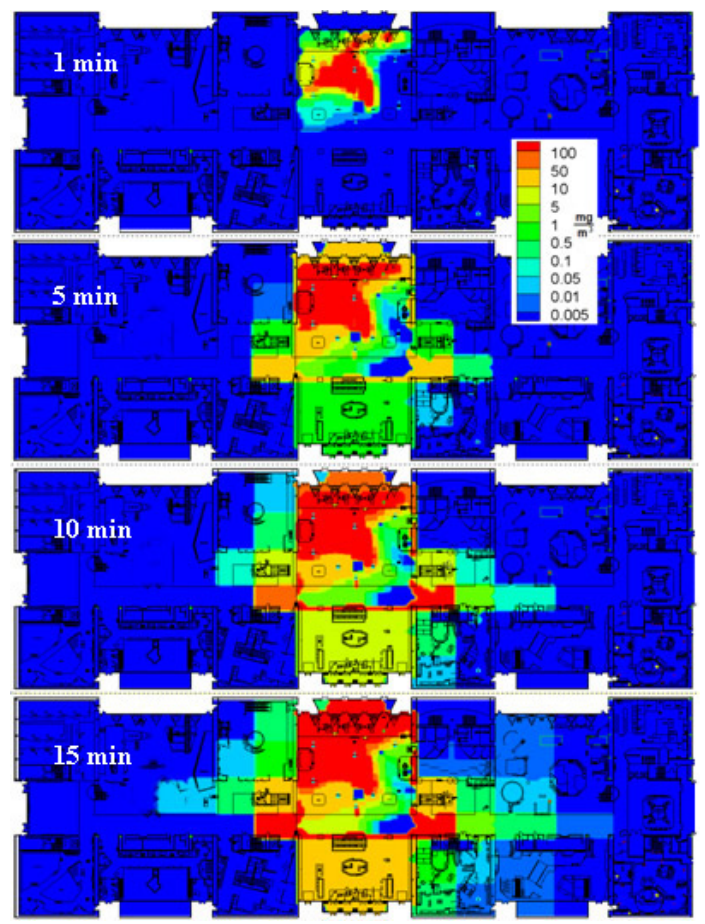

Figure 4: $\quad$ Toxic nerve agent is dispersed into a large facility.

Three scenarios were run and the end state of the occupants determined (Dead, Incapacitated, Sick and Minimal Effect). The people were then plotted in their original locations (Figures 5 and 6).

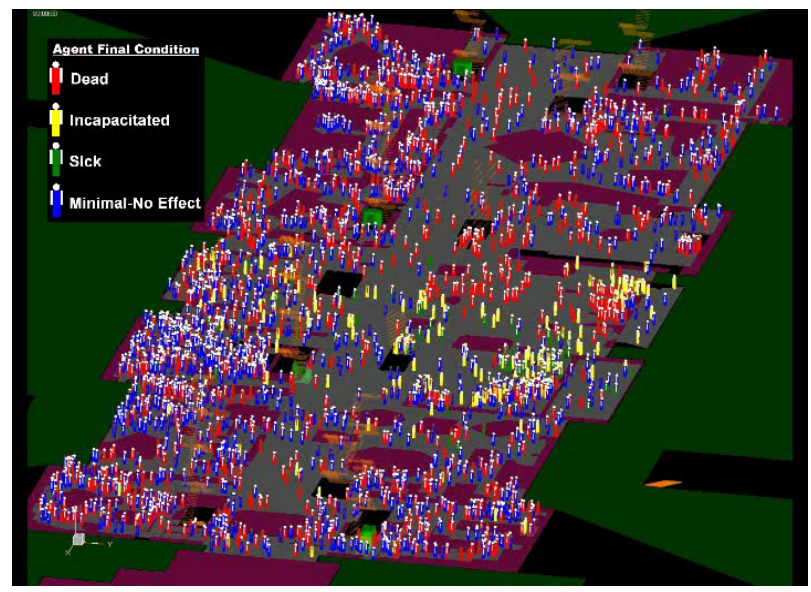

Figure 5: Scenario with 5,000 people in a facility attacked with Sarin Gas (Scenario A). 
In Scenario "A", no announcement was made via the building's public announcement system. Occupants had to either observe the event and decide to evacuate or they had to learn this information from others in the building and

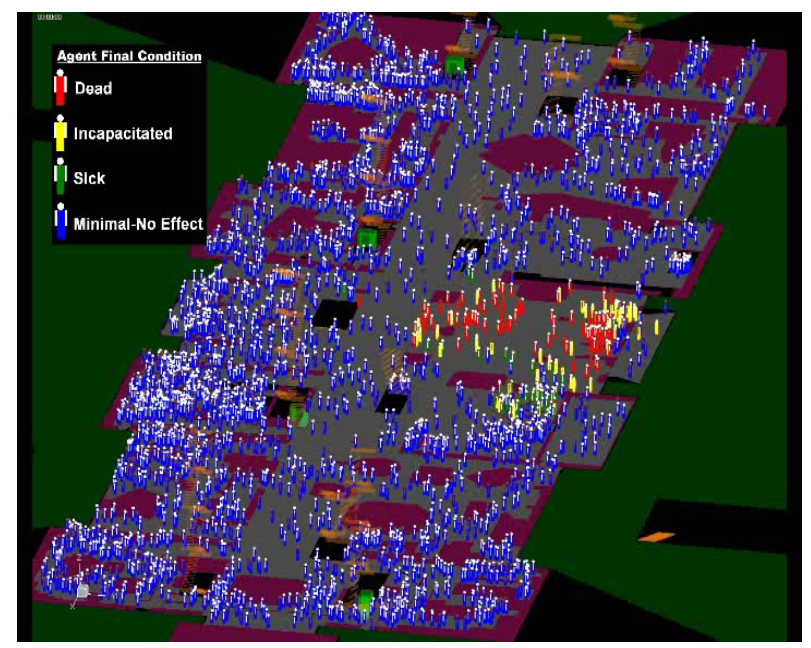

Figure 6: Scenario with 5,000 people in a facility attacked with Sarin Gas (Scenario C).

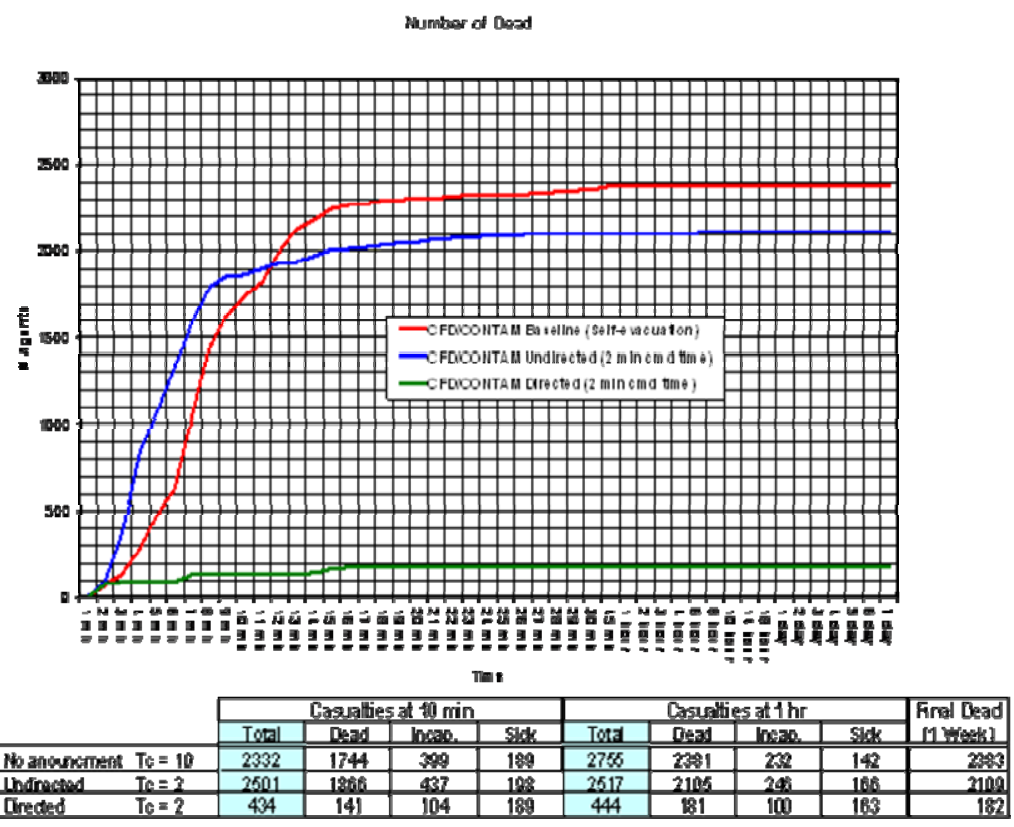

Figure 7: $\quad$ Casualties versus time for Scenario A, B and C. 
then self-evacuate. In this case, most of the simulated humans replicated typical human behaviour and attempted to exit through the main lobby (i.e., the way they came into the facility). In this case, 2383 people died (Figure 5).

In Scenario "B", a general announcement was made two minutes after the event that simply stated "evacuate the facility" with no further instructions. In this case, most people still tried to evacuate through the main entrance even though there were many closer emergency exits. In this case, 2109 people died.

Finally, in Scenario "C" an announcement was made two minutes after the release of the gas that stated, "This is an emergency, exit immediately through the nearest exit, do not approach the main lobby." This set of simple instructions saved many lives. In this case only 183 people were predicted to die (Figure 6). The results from all three scenarios are summarized in Figure 7.

\subsection{Active shooters in a highly secure facility}

In contrast to the previous example that involved thousands of people, this example shows a much smaller group of agents. Three well armed attackers attempt to enter the secure facility using explosives (Figure 8). They are also well armed with guns. In this scenario, the perimeter sensors alert three well trained security guards inside of the facility. Multiple variations can then be run to examine such things as 1) what if I had more guards; 2) what if there were improved blast and ballistic resistant areas within the facility; 3) what if the guards are not in position or take longer to respond than required by their training; 4) what if there are bystanders in the facility that become hostages; and 5) what if the delay times on the security systems were improved through the use of newer technology. Running such parametric studies allow the users to visualize and examine potential outcomes that would not be possible otherwise.

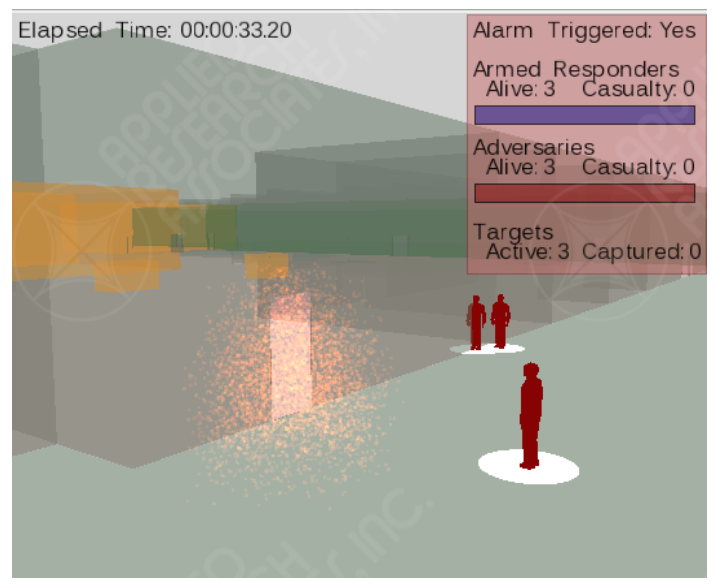

Figure 8: Armed attackers use explosives to enter a highly secure facility. 


\section{Conclusion}

As man-made as well as naturally occurring disasters and attacks happen with greater frequency and consequence, the responsibility to improve our knowledge, planning, and preparation for these events becomes ever more urgent. Emergency responders and planners cannot rely on traditional exercises and historical analysis for accurate situational outcomes without becoming subject to the many generalizations and assumptions upon which these methods are based. Previous modeling and simulation methods have likewise been based on historical precedence, limited assumptions and generalizations that result in inaccurate outcomes. As a society, we cannot continue to plan exclusively for the "last event" as is typically the case after a major catastrophic attack occurs. These methods do not arm decision makers with the correct, detailed information they require for accurate analysis and response preparedness. Our society must be more proactive and forward-thinking in evaluating innovative responses to attacks in order to stay one step ahead of the next perpetrator. Such ambitious endeavours can only be accomplished through the appropriate utilization of high fidelity modeling.

Therefore, it is now more important than ever to effectively utilize state-ofthe-art cognitive task analysis and human agent modeling technologies to develop the most accurate, comprehensive and realistic scenario data sets. We must then apply these data sets to better prepare for armed attackers and to successfully defend our citizens. As modeling and simulation systems and cognitive task analysis science further advance and integrate, detailed virtual investigations will continue to serve an even greater role in planning effective response and recovery operations for shootings, attacks, and other mass casualty events. 